\title{
Cross-Infection Control of Coronavirus Disease 2019 at the Emergency Department in Taiwan
}

\author{
Chien-Fu Lin ${ }^{1} \cdot$ Kuan-Han Wu ${ }^{1} \cdot$ I-Min Chiu ${ }^{1,2}$ (D) \\ Received: 25 June 2020 / Accepted: 15 July 2020 / Published online: 27 July 2020 \\ (C) Springer Science+Business Media, LLC, part of Springer Nature 2020
}

The coronavirus disease 2019 (COVID-19) pandemic has, as of today, spread in at least 200 countries and killed more than 470,000 people. To date, Taiwan has had 447 confirmed cases and ranks 148th on the list of infected areas. Most importantly, in Taiwan, there have been no health care professionals (HCPs) in the emergency department (ED) confirmed with COVID-19 to date.

In EDs, HCPs must be careful about contagious respiratory agents not only from direct patient contact but also potential viral shedding from other members. Cross-contamination among coworkers in the ED can occur through various methods and occasionally poses a bigger threat compared to patient examination [1,2]. Droplet transmission can take place in the working environment and rest area. At Kaohsiung Chang Gung Memorial Hospital, a medical center in Taiwan with previous experience of a hospital cluster outbreak of the severe acute respiratory syndrome (SARS) epidemic in 2003 [3], we had developed preventive strategies following detailed steps to control cross-infection of COVID-19 for frontline HCPs in the ED.

1. TOCC self-screening questionnaire: HCPs should assess and record their own symptoms and travel, contact, and cluster histories into the websites every week before starting work.

This article is part of the Topical Collection on Systems-Level Quality Improvement

I-Min Chiu

ray1985@cgmh.org.tw; outofray@hotmail.com

1 Department of Emergency Medicine, Kaohsiung Chang Gung Memorial Hospital, No. 123, Dapi Rd. Niaosong Dist, Kaohsiung City 83301, Taiwan

2 Department of Computer Science and Engineering, National Sun Yet-Sen University, Kaohsiung City, Taiwan
2. Regular body temperature monitoring: Body temperature checkpoints should be set at every entrance of the ED. Further, every HCP should record his/her own temperature into the hospital record system every day.

3. Complete personal protective equipment (PPE): Every first-line clinical HCP should wear PPE, including helmets, gloves, face shields, and N95 masks, before entering ED.

4. Outdoor ED triage and isolated waiting room: Outdoor COVID-19-specific clinics with isolated testing equipment and waiting room should be set-up for separating other ED patients without specific symptoms or a TOCC history from those suspected with the COVID-19 infection. Showering rooms outside the isolated clinics should also be set-up for HCPs to be used after the duty.

5. Separating the work and rest areas: As shown in fig. 1, for the designated HCPs in charge of suspected COVID19 patients, not only isolated clinics but also independent rest areas should be planned for preventing possible transmission among colleagues. Even in the same rest area, employees should maintain a 2-m distance from each other while dining or taking a short break. Every table in the dining area and office rooms should be separated with an acrylic board in case of droplet or contact transmission. Furthermore, all areas, including offices, bathrooms, common areas, and shared electronic equipment, should be cleaned and disinfected routinely at the end of every shift.

In addition, PPE should be removed step-by-step at check points to prevent possible colonization transmitted through its surface. The key to successfully arresting the spread of the COVID-19 epidemic is adherence to standard protocols in daily practice. We hope these experiences related to COVID-19 could help set up an epidemic prevention system and prevent widespread panic in EDs. 
Fig. 1 Conceptual scheme of traffic control on work and rest area separation

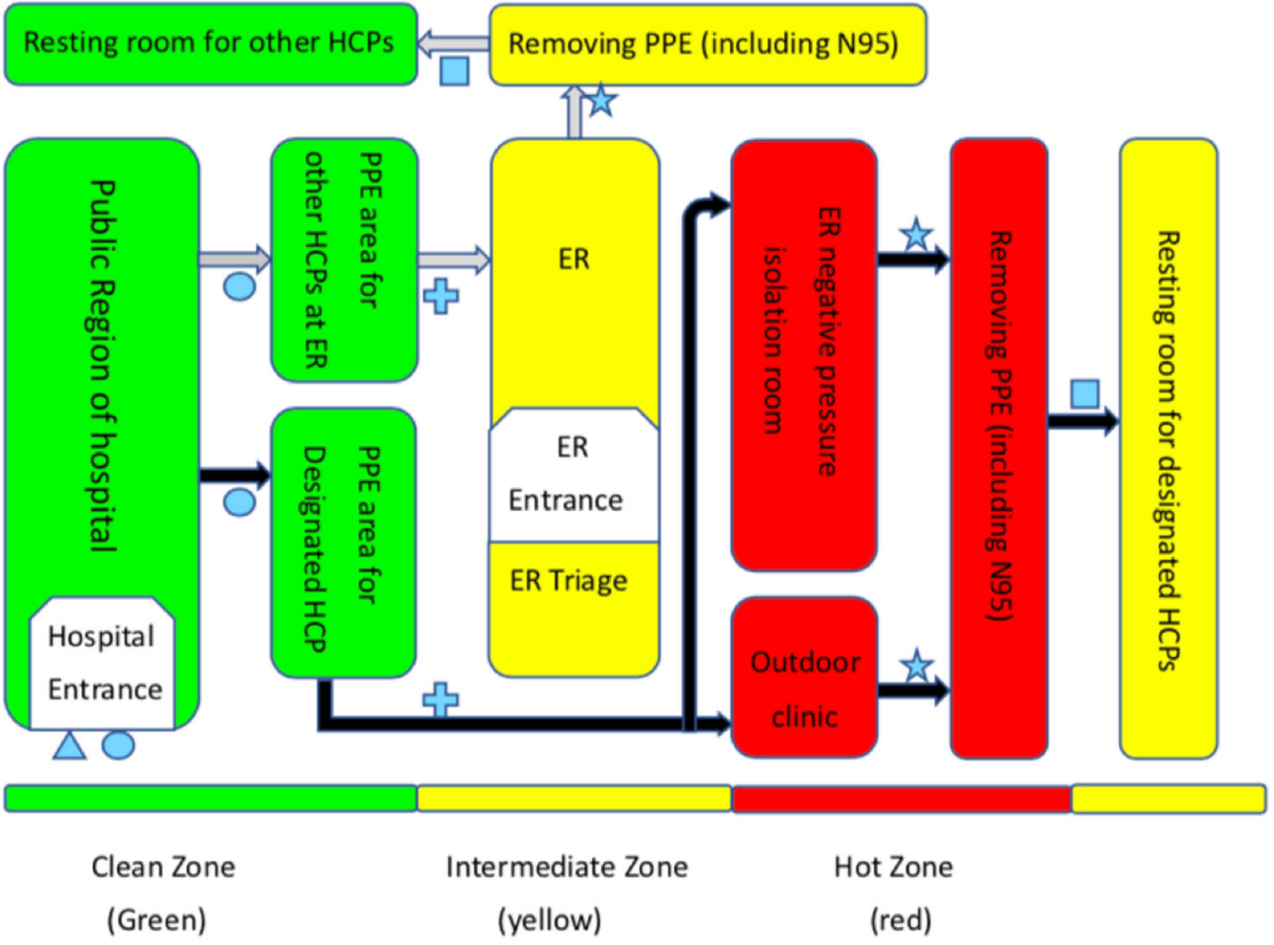

\begin{tabular}{|c|c|}
\hline Icon & Meaning \\
\hline$\bigcirc$ & Check Point: Hand disinfection \\
\hline$\hat{\imath}$ & Check Point: Decontamination(removing all PPE), Hand disinfection \\
\hline$\square$ & Check Point: Surgical mask wearing, Hand disinfection \\
\hline$\triangle$ & Check Point: Surgical mask wearing, Hand disinfection \\
\hline گુ & Check Point: N95 mask wearing, Hand disinfection \\
\hline$\Longrightarrow$ & Pathway: Other HCPs of emergency department \\
\hline & Pathway: Designated HCPs for COIVD 19 suspects of emergency department \\
\hline
\end{tabular}

\section{Compliance with ethical standards}

Conflict of interest Authors declare that we have no conflict of interest.

Ethical approval This article does not contain any studies with human participants or animals performed by any of the authors.

\section{References}

1. Rothman RE, Irvin CB, Moran GJ, et al. Respiratory hygiene in the emergency department. Ann Emerg Med. 2006;48(5):570-582.
2. Dancer SJ. Controlling hospital-acquired infection: focus on the role of the environment and new technologies for decontamination. Clin Microbiol Rev. 2014;27(4):665-690.

3. Chen WK, Wu HD, Lin CC, Cheng YC. Emergency department response to SARS, Taiwan. Emerg Infect Dis. 2005;11(7):10671073.

Publisher's note Springer Nature remains neutral with regard to jurisdictional claims in published maps and institutional affiliations. 